\title{
Intra-individual response variability assessed by ex-Gaussian analysis may be a new endophenotype for attention-deficit/hyperactivity disorder
}

\section{Marcela Patricia Henríquez-Henríquez ${ }^{1,2}$, Pablo Billeke ${ }^{2,3}$, Hugo Henríquez ${ }^{4}$, Francisco Javier Zamorano ${ }^{2,3}$, Francisco Rothhammer ${ }^{5}$ and Francisco Aboitiz ${ }^{2}$}

1 Department of Clinical Laboratories, Pontificia Universidad Católica de Chile, Santiago, Chile

${ }^{2}$ Cognitive Neurosciences Laboratory, Department of Psychiatry, Pontificia Universidad Católica de Chile, Santiago, Chile

${ }^{3}$ Centro de Investigación en Complejidad Social (CICS), Facultad de Gobierno, Universidad del Desarrollo, Santiago, Chile

${ }^{4}$ Medical Technology School, Universidad Mayor, Santiago, Chile

${ }^{5}$ Instituto de Alta Investigación, Universidad de Tarapacá, Arica, Chile

\author{
Edited by: \\ John Vijay Sagar Kommu, National \\ Institute of Mental Health and \\ Neurosciences, India \\ Reviewed by: \\ Preeti Jacob, National Institute of \\ Mental Health and Neurosciences, \\ India \\ Sowmya Bhaskaran T. S., National \\ Institute of Mental Health and \\ Neurosciences, India \\ *Correspondence: \\ Marcela Patricia \\ Henríquez-Henríquez, Department of \\ Clinical Laboratories, Pontificia \\ Universidad Católica de Chile, Vicuña \\ Mackena 4686, Santiago 7820436, \\ Chile \\ e-mail:mhenri@med.puc.cl
}

Intra-individual variability of response times (RTisv) is considered as potential endophenotype for attentional deficit/hyperactivity disorder (ADHD). Traditional methods for estimating RTisv lose information regarding response times (RTs) distribution along the task, with eventual effects on statistical power. Ex-Gaussian analysis captures the dynamic nature of RTisv, estimating normal and exponential components for RT distribution, with specific phenomenological correlates. Here, we applied ex-Gaussian analysis to explore whether intra-individual variability of RTs agrees with criteria proposed by Gottesman and Gould for endophenotypes. Specifically, we evaluated if normal and/or exponential components of RTs may (a) present the stair-like distribution expected for endophenotypes (ADHD > siblings > typically developing children (TD) without familiar history of ADHD) and (b) represent a phenotypic correlate for previously described genetic risk variants. This is a pilot study including 55 subjects (20 ADHD-discordant sibling-pairs and 15 TD children), all aged between 8 and 13 years. Participants resolved a visual Go/Nogo with 10\% Nogo probability. Ex-Gaussian distributions were fitted to individual RT data and compared among the three samples. In order to test whether intra-individual variability may represent a correlate for previously described genetic risk variants, VNTRs at DRD4 and SLC6A3 were identified in all sibling-pairs following standard protocols. Groups were compared adjusting independent general linear models for the exponential and normal components from the ex-Gaussian analysis. Identified trends were confirmed by the non-parametric JonckheereTerpstra test. Stair-like distributions were observed for $\mu(p=0.036)$ and $\sigma(p=0.009)$. An additional "DRD4-genotype" $\times$ "clinical status" interaction was present for $\tau(p=0.014)$ reflecting a possible severity factor. Thus, normal and exponential RTisv components are suitable as ADHD endophenotypes.

Keywords: ex-Gaussian analysis, ADHD, intra-individual variability, endophenotypes, response time

\section{INTRODUCTION}

Symptom-based diagnostic systems such as the current versions of the Diagnostic and Statistical Manual of Mental Disorders (DSM) (1) and the International Classification of Diseases (ICD) (2) have proved to be useful in the clinical approach to the neuropsychiatric patient and in epidemiological settings. Notwithstanding, in opinion of some authors, their massive utilization in moleculargenetic studies has probably hampered (or even obstructed) the identification of susceptibility genes for neuropsychiatric disorders, due to the inherent multidimensionality of phenotypes defined by them (3). This multidimensionality probably hides genetic heterogeneity, non-genetic phenocopies, and complex networks of gene-gene and gene-environment interactions, among other confounding phenomena, lowering the statistical power of association studies (3-5).
Taking this into account, it has been proposed to replace symptom-based phenotypes by quantifiable markers of liability or "risk" for a specific disorder (4). These markers, generically called "endophenotypes" constitute a more "direct" expression of the gene effect, since they conceptually lie between the gene and the disorder and - in consequence - are influenced by fewer genetic and environmental variables than the disorder itself. In addition to their potential role improving the statistical power of molecular-genetic studies, endophenotypes should be a valuable tool when studying how the already-known genetic risk variants are related to the neurobiological and neuro-physiological phenotypes that underlie psychiatric disorders, which could be the first step to elucidate the specific domains of brain function influenced by these variants. This kind of approach has been successfully applied, for example, in the functional char- 
acterization of risk genetic variants for psychotic and affective disorders (6-8).

In the last few years, researchers have started to explore the potential of some neuro-cognitive and electrophysiological/radiological markers as endophenotypes for attentional deficit/hyperactivity disorder (ADHD). Among them, intraindividual variability [defined as short-term changes in behavior that are signed as moment-to-moment fluctuations in task performance $(9,10)]$ seems to be especially promising. Elevated intra-individual variability of the response times (RTisv) in ADHD patients is one of the most consistent findings across the ADHD literature and has been documented in task assessing working memory (11), attention $(12,13)$, inhibitory control $(12,14)$, and choice discrimination (15). Interestingly, the magnitude of the between-group differences reported tends to be larger than most other neuropsychological parameters studied to the date (16). Additionally, intra-individual variability is in close agreement with some of the criteria proposed by Gottesman and Gould to facilitate the exclusion of spurious endophenotypes and to increase the likelihood of identifying stronger associations with genetic factors $(4,17)$. In this context, it is worth to mention two important lines of evidence: (1) studies showing that affected relatives of ADHD children present greater intra-individual variability compared to controls $(18,19)$ and $(2)$ studies supporting the heritability of intra-individual variability $(15,18,20)$.

Traditionally, intra-individual variability has been estimated by collapsing responses across the entire time interval of the task, resulting in a single point parameter such as the standard deviation of the response time (RTSD) or the coefficient of variation of the response time (RTCV), which should capture and represent how data spread around the mean value. This method implies to lose a significant amount of specific information regarding the distribution of response times (RTs) along the task, with eventual effects on statistical power when comparing RTisv among clinical groups. Particularly, this method does not recognize the asymmetrical positive skew that occasional lapses in attention are expected to produce in the RTs distributions (21).

During the last decade, the field has moved toward new statistical approaches aiming to obtain more complete and specific characterizations of the RTisv. Among these approaches, the exGaussian analysis on the RTs has provided enlightening results regarding the dynamic nature of intra-individual variability in ADHD. Basically, the ex-Gaussian analysis is based on the convolution of an exponential and a Gaussian function, obtaining three parameters: $\mu(\mathrm{mu})$, corresponding to the mean of the normal component, $\sigma$ (sigma), corresponding to the SD of the normal component, and $\tau$ (tau), which describes the mean of the exponential component. When fitting ex-Gaussian distributions to RT data, greater values for $\tau$ indicate a higher frequency of excessively long RTs (22). In general, most of the studies that have applied ex-Gaussian analysis on RT data from ADHD patients showed elevation of $\tau$ component in the ADHD group (11,22-27). This finding is consistent with lapses in attention, due to a defective effort control mechanisms (22).

In this work, we hypothesize that RTisv - expressed as the $\tau$ and $\sigma$ components of the ex-Gaussian distribution of RTs - may be a potential endophenotype for ADHD. In order to address this hypothesis, we first explored whether healthy siblings of ADHD patients may present intermediate values of intra-individual variability in front of a motor inhibition task (Go/NoGO) when compared to ADHD patients and typically developing children (TD) without family history of ADHD, and so that, whether intra-individual variability may follow the hypothetical distribution postulated for endophenotypes. As a second step, we explored whether RTisv may represent a phenotypic correlate for previously described genetic risk variants for ADHD in the genes encoding for the dopaminergic receptor $\mathrm{D} 4$ (DRD4) and for the dopaminergic transporter 1 (SLC6A3/DAT1). We choose the aforementioned genetic risk variants based on previous evidence suggesting that RTisv may be associated to dopamine dysfunction (28-30).

To the best of our knowledge, this is the first study applying the ex-Gaussian approach to simultaneously characterize the performance of ADHD patients, their asymptomatic first degree relatives, and TD without family history of ADHD. Additionally, after an exhaustive search, we did not find other studies applying ex-Gaussian analysis in order to evaluate intra-individual variability as a phenotypic correlate to genetic variants in DRD4 and SLC6A3/DAT1 genes, which has been previously linked to ADHD.

\section{MATERIALS AND METHODS}

\section{PARTICIPANTS}

In order to evaluate eventual differences in regards to the RT distribution observed among ADHD patients, their non-affected siblings, and TD without family history of ADHD, we evaluated a total of 55 subjects, corresponding to 20 discordant sibling-pairs and 15 unaffected children from the general population. Sibling-pairs were originally recruited as part of a parallel genetic association study started in 2003 (31). This study included families from the Great Santiago Area, referred from general psychiatric and neurological outpatient services directed to medium-income population. Unaffected children without family history of ADHD were recruited from a medium-income school from the same socio-economical and geographical area. Cases and controls belong to a narrow age-range (ranged between 8 and 13 years) in order to avoid age-confounding effects on performance/neurobehavioral measurements. Groups were comparable by age distribution (ADHD group: mean $=11.2$ years, $\mathrm{SD}=2.47$; sibs group: mean $=11$ years, $\mathrm{SD}=2.35$; unaffected children from general population: mean $=11.6$ years, $\mathrm{SD}=0.9$ $F=0.25 ; p=0.77$ ) and gender distribution [ADHD group: 17 boys and 3 girls; sibs group: 13 boys and 7 girls; unaffected children from general population: 9 boys and 6 girls $\chi^{2}(2)=3.1 ; p=0.21$ ]

Inclusion criteria for ADHD children were as follows: ADHD combined subtype according to DSM (32), age between 8 and 13 years, having at least one unaffected sibling in the same range of age and good response to stimulant medication. We included the latest criteria in order to reduce clinical heterogeneity in our size-restricted sample. Operationally, we defined "good response to medication" as a clinically significant improvement in symptomatology reported by parents in an interview with a competent specialist and documented as at least $20 \%$ reduction in the Conners' Abbreviated Parent-Teacher Questionnaire for ADHD symptoms (33). All patients included in this study were treated with $\mathrm{D}$-amphetamine or methylphenidate, both in doses ranging 
10-30 mg/day. A wash-out period of $24 \mathrm{~h}$ free of medication was required before the neuropsychological assessment.

In the case of the groups composed by non-affected siblings and TD from the general population, inclusion criteria were age between 8 and 13 years old and absence of ADHD or any other psychiatric morbidity according to DSM-IV criteria. Additionally, a negative familiar history of ADHD was required in the TD group. Familiar history of ADHD was explored in first degree relatives (siblings and parents) of TD participants by means of a semistructured interview assessing presence/absence of DSM-IV criteria during childhood. Children with neurological deficit at physical examination and/or abnormal baseline electroencephalography (EEG) were excluded from the study. All aforementioned inclusion/exclusion criteria were assessed according to current ADHD diagnosis guidelines by a competent specialist (child psychologist or child neurologist).

We only included ADHD patients and their unaffected siblings for genotyping analysis. This design was chosen in order to control for an eventual population stratification effect. As additional advantage, discordant sibling-pairs share most of the psychosocial and familial factors that might interact with a potential genetic predisposition. Thus, for the genetic part of the study, 40 participants (corresponding to 20 siblings-pairs) were assigned to "Risk" or "Non-Risk" genotype groups accordingly with the criteria described in Section "Group Comparisons."

\section{GENOTYPING}

Genomic DNA was isolated from peripheral blood lymphocytes by standard methods and amplified by polymerase chain reaction (PCR) to identify the VNTR of the DRD4 and SLC6A3 loci according to the protocols described by Lichter et al. (34) and by Cook et al. (35), respectively. PCR products were visualized by electrophoresis in 3\% agarose gels. Details on the PCR primers sequences have been previously reported by our group (36).

\section{TASK AND PROCEDURES}

Details on the Go-NoGo task performed in this study have been previously reported by our group (36). Briefly, all participants responded to an $8 \mathrm{~min}$ long Go-NoGo Task with a Go:NoGo rate of 9:1. Stimuli corresponded to green (Go) and red (NoGo) circles of $2 \mathrm{~cm}$ in diameter presented at the center of a black screen during $300 \mathrm{~ms}$, with an inter-trial period of $1,000 \mathrm{~ms}$. A pseudorandom process ensured that at least six Go stimuli were presented before each NoGo stimulus. The total task comprised the presentation of 360 Go and 40 NoGo stimuli. The instruction was to click in a console as fast as possible, with the dominant hand, after every Go stimulus, but to inhibit the response in front of NoGo stimuli. A treatment wash-out period of $24 \mathrm{~h}$ was required in all ADHD patients previous to task performance. No additional tasks or procedures were performed in the same session. No exclusions due to task non-compliance were registered.

\section{DATA ANALYSIS}

\section{Data treatment for ex-Gaussian analysis}

Data corresponding to the response time (RT) in front of all Go stimuli were obtained from each subject. Only correct responses were computed in order to determine the parameters of the
ex-Gaussian distribution of RT, according to the data-analysis protocol originally described by Leth-Stevenson et al. (22). Additionally, any single RT of $<100 \mathrm{~ms}$ was considered as accidental key press and was excluded. Ex-Gaussian distributions were fitted to RT data using the $R$ statistical package (37), and $\mu, \sigma$, and $\tau$ parameters were computed for each participant. The number of RT observations used for each ex-Gaussian fit depended on the accuracy of responding ( mean $=287$ trials, range $=97-357$ ). Finally, in order to assess the Goodness-of-fit of the ex-Gaussian models, the empirical distribution for each child was compared to a random ex-Gaussian distribution using the values for $\mu, \sigma$, and $\tau$ adjusted for the same subject. Comparison was performed by means of the Kolmogorov-Smirnov Test. There were no significant differences between empirical and ex-Gaussian distributions for any one of the analyzed subjects.

\section{Group comparisons}

In order to compare the characteristics of ex-Gaussian distributions among ADHD children, their non-affected sibs, and TD from the general population (TD), we adjusted independent general linear models for $\mu, \sigma$, and $\tau$, using age and sex as co-variates and "clinical group" (ADHD, non-affected sib, or TD) as an ordinal variable, with ADHD as the highest ordinal value and TD as the lowest. Additionally, we confirmed the statistical significance of the detected trends by means of the non-parametric JonckheereTerpstra test to evaluate trend in the data (e.g., ADHA > nonaffected sibs $>$ TD). Significance was defined as alpha $=0.05$. For multiple comparisons, $p$-values after post hoc analysis are reported.

When comparing the characteristics of the ex-Gaussian distributions regarding to the presence/absence of genetic risk variants previously linked to ADHD in the DRD4 and SCL6A3 genes, on the other hand, we applied three consecutive approaches. First, eventual differences in $\mu, \sigma$, and $\tau$ between the genetic risk and non-risk groups were preliminary explored by means of non-parametrical methods (Wilcoxon Rank Test). In a second step, significance levels were adjusted by age, sex, and clinical status by means of general linear models. Finally, the effect of an interaction between the "clinical status (presence/absence of ADHD) and genotype group (risk/control) was explored by general linear models for $\mu, \sigma$, and $\tau$, with the independent variables "genetic risk group" (risk versus control group), age, sex, clinical status, and "genetic risk group $\times$ clinical status" interaction. As mentioned above, in order to control for population stratification, only sib-pairs were included for genetic analysis.

"Genetic risk groups" were defined as follows: (a) For DRD4; the "control" or "non-risk" group was comprised by homozygous for the DRD4 4-repeat allele. A total of 22 subjects were assigned to this group and 9 of them were ADHD patients. The "risk" group, on the other hand, was constituted by carriers of at least one copy of alleles $7 \mathrm{R}$ ( $n=11 ; 3$ of them in homozygous state) or $2 \mathrm{R}$ ( $n=7$; two of them in homozygous state). This group included 18 subjects and 11 subjects presented ADHD among them. There was no association between allele possession for Exon 3 VNTR of DRD4 and clinical status $\left[\chi^{2}(1)=0.9, p=0.34\right]$. We chose to include carriers of the $7 \mathrm{R}$ and $2 \mathrm{R}$ alleles in the "risk group" based on previous reports of association between ADHD and these variants in different populations (38-40). Additionally, some in vitro studies 
have suggested that receptors encoded by $2 \mathrm{R}$ and $7 \mathrm{R}$ alleles mediate a reduced response to dopamine in comparison with the $4 \mathrm{R}$ allele (41). (b) For SLC6A3; the "risk group" were comprised by homozygous for $10 \mathrm{R}$ ( $n=24 ; 13$ of them were ADHD patients) and the "control" or "non-risk" was constituted by subjects with any other genotype ( $n=15$; 6of them were ADHD patients). The latter group comprised 14 participants with 9R/10R genotype and 1 homozygous for the 9R allele. There was no association between allele possession for $3^{\prime}$ UTR VNTR of SLC6A3 and clinical diagnosis of $\mathrm{ADHD}, \chi^{2}(1)=0.28, p=0.59$.

\section{ETHICAL ISSUES}

All procedures performed as part of this study were approved by the Ethics Committee of the Pontificia Universidad Catolica de Chile. The study was fully explained to children and their parents, and they both agreed to participate by signing written consent forms.

\section{RESULTS}

\section{COMPARISON OF EX-GAUSSIAN DISTRIBUTIONS AMONG ADHD CHILDREN, THEIR NON-AFFECTED SIBS, AND TD FROM THE GENERAL POPULATION}

Figure 1 shows the graphical representation and values for $\mu, \sigma$, and $\tau$ when adjusting ex-Gaussian distributions to the RT data from ADHD patients (solid gray line), non-affected sibs (dotted line), and TD without family history of ADHD (solid black line). In order to statistically explore eventual differences among these groups, we performed linear models for the aforementioned parameters, with clinical group as the ordinal variable. Results from this analysis are summarized in Table $\mathbf{1}$. There was a statistically significant effect of the independent variable "clinical group" on the parameters $\mu$ (corresponding to the mean of the Gaussian/normal component of the distribution; $p$-value $=0.015$ ). Since models were adjusted with ADHD as the highest ordinal value and TD as the lowest, we can assume that $\mu$ presented with the stair-like distribution typically expected for endophenotypes. A similar interesting trend was observed for $\sigma$ (corresponding to the SD of the Gaussian/normal component), although it did not reach statistical significance after controlling for age and sex $(p$-value $=0.052)$. We did not find a statistically significant effect for "clinical group in the models adjusted for $\tau$. Similar results were obtained using Jonckheere-Terpstra test to evaluate the trend $\mathrm{ADHD}>$ non-affected sibs $>\mathrm{TD}(\sigma: \mathrm{JP}=587, p$-value $=0.009$, predictive strength $r=0.35 ; \mu$ : JP $=559, p$-value $=0.0358$, predictive strength $r=0.36 ; \tau$ : $\mathrm{JP}=523, p$-value $=0.13$ ).

\section{COMPARISON OF EX-GAUSSIAN DISTRIBUTIONS REGARDING TO THE PRESENCE/ABSENCE OF GENETIC VARIANTS PREVIOUSLY LINKED TO ADHD IN THE DRD4 AND SCL6A3 GENES}

Figure 2 shows the RT distributions obtained when we classified and analyzed the subjects in regards to their "genotype group" for DRD4 (2A) and SCL6A3 (2B). In the case of DRD4, subjects were classified depending on the presence/absence of VNTR variants previously linked to ADHD in the exon III of DRD4, with carriers of at least one copy of the $7 \mathrm{R}$ or $2 \mathrm{R}$ allele considered as the risk group and homozygous for $4 \mathrm{R}$ as the controls/non-risk group. The ex-Gaussian distribution adjusted among subjects from the genetic risk group is more skewed than the ex-Gaussian distribution for DRD4-4R homozygous, which is expressed as a bigger value for $\tau$ (unadjusted $p=0.013$; see

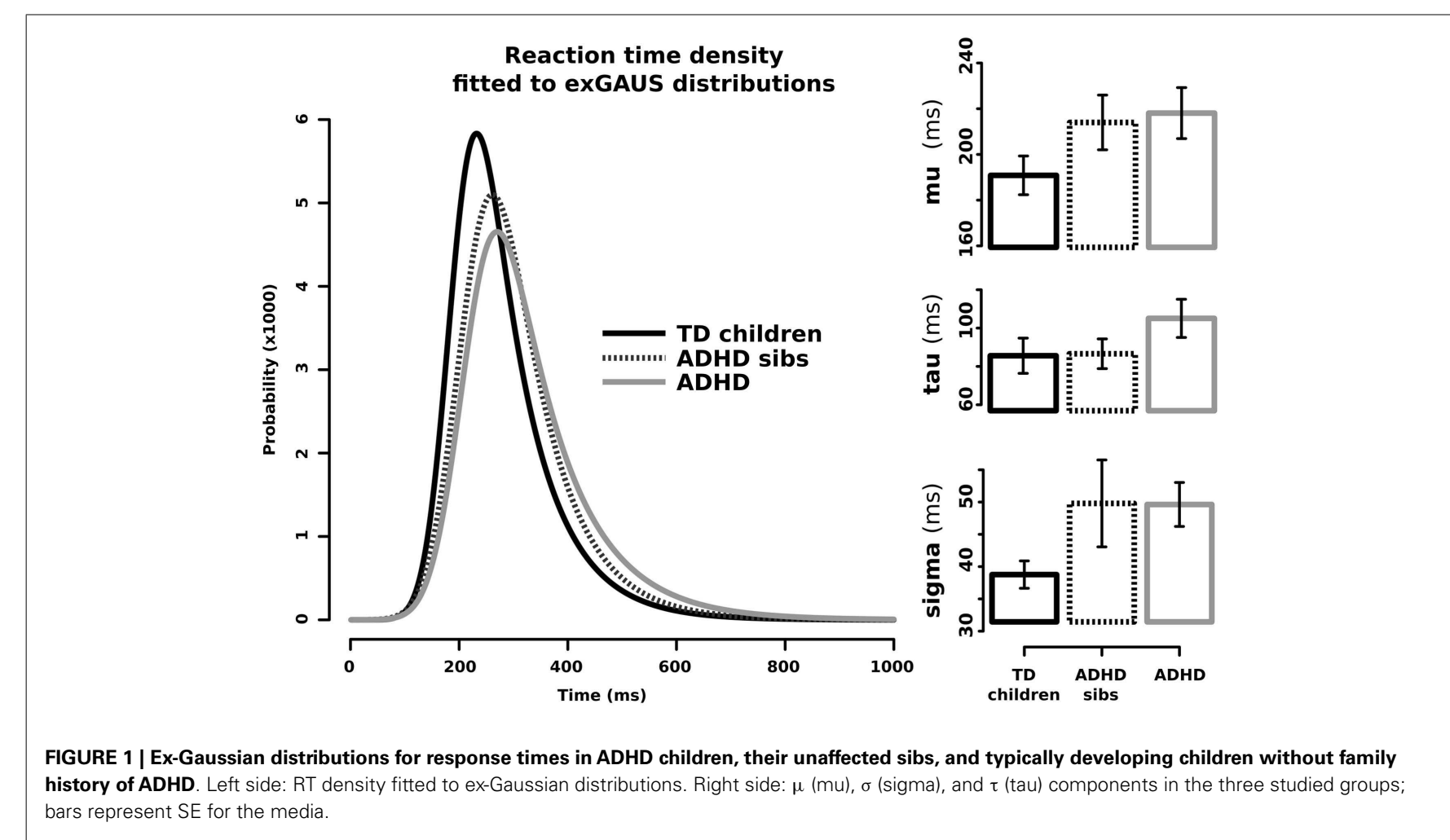


Table 1 | Summary of estimated effects in general linear models adjusted for mu, sigma, and tau parameters

\begin{tabular}{lllcccc}
\hline $\begin{array}{l}\text { Model } \\
\text { for... }\end{array}$ & $\begin{array}{l}\text { Independent } \\
\text { variable }\end{array}$ & $\begin{array}{l}\text { Estimated } \\
\text { effect }\end{array}$ & SE & $\boldsymbol{t}$ & Effect-size $\boldsymbol{r}$ & $\boldsymbol{p}$-value \\
\hline$\mu$ & Clinical group & 19.09 & 7.61 & 2.5 & 0.33 & 0.015 \\
& Age & -6.96 & 2.88 & -2.41 & 0.31 & 0.019 \\
& Sex & -28.82 & 13.28 & -2.16 & 0.28 & 0.035 \\
$\sigma$ & Clinical group & 6.33 & 3.19 & 1.99 & 0.26 & 0.052 \\
& Age & -3.16 & 1.21 & -2.61 & 0.34 & 0.011 \\
& sex & -9.32 & 5.56 & -1.65 & - & 0.1 \\
$\tau$ & Clinical group & 3.67 & 7.74 & 0.52 & - & 0.6 \\
& Age & -7.45 & 2.93 & -2.5 & 0.33 & 0.015 \\
& sex & -2.8 & 13.5 & -0.21 & - & 0.83 \\
\hline
\end{tabular}

Table 2 for a summary of comparisons between DRD4/SCL6A3 genotype groups). Interestingly, linear models demonstrated a statistically significant effect of the "genotype group" $x$ "clinical status" interaction exclusively on $\tau$ ( $p$-value $=0.014$; see Table 3 for a summary of interaction models). This interaction reflects a greater $\tau$ among ADHD subjects carrying DRD4-risk alleles in comparison to DRD4-4R homozygous (Kruskal-Wallis rank sum test, $\chi^{2}=0.9562, \mathrm{df}=1, p$-value $=0.01467$, effect-size $r=0.44$, and post hoc p-value: 0.03196; see Figure 3). In the case of SCL6A3, on the other hand, we did not observe any significant effect of genotype on any of the ex-Gaussian parameters analyzed (see Figure 2; Table 2).

\section{DISCUSSION}

In the present study, we hypothesized that RTisv, assessed by means of the ex-Gaussian approach, is suitable as an endophenotype for ADHD. The principal aim for the first part of the study was to explore whether any of the parameters characterizing the exGaussian curves fitted for ADHD patients, their healthy siblings, and/or TD children without family history of ADHD, present the "stair-like distribution" expected for an ADHD endophenotype. In the second part, we intended to explore if the parameters characterizing the ex-Gaussian distribution of RTs may correspond to phenotypic correlates for genetic variants in DRD4 and SCL6A3/DAT1, which have been previously linked to ADHD.

From a phenomenological perspective, ex-Gaussian analysis is able to distinguish whether differences in group performances obey to (a) a generalized slowing down of the responses times (reflected at the $\mu$ component), (b) an increased variability throughout the complete distribution (reflected at the $\sigma$ component), or (c) an increased number of abnormally slow responses (reflected at the $\tau$ component). The aforementioned distinction could be mechanistically relevant, since it has been suggested that larger $\tau$ values may reflect inconsistent effort and fluctuations in attention (attentional lapses), while larger $\sigma$ components may reflect deficits in motor timing and/or impaired response preparation $(22,25)$. Notwithstanding the potential enclosed in the aforementioned fine-grained analysis, to date, only few studies have applied this approach to explore the characteristics of responses of ADHD children. The majority of these studies have

\section{Density fitted to exGAUS distribution per subject}
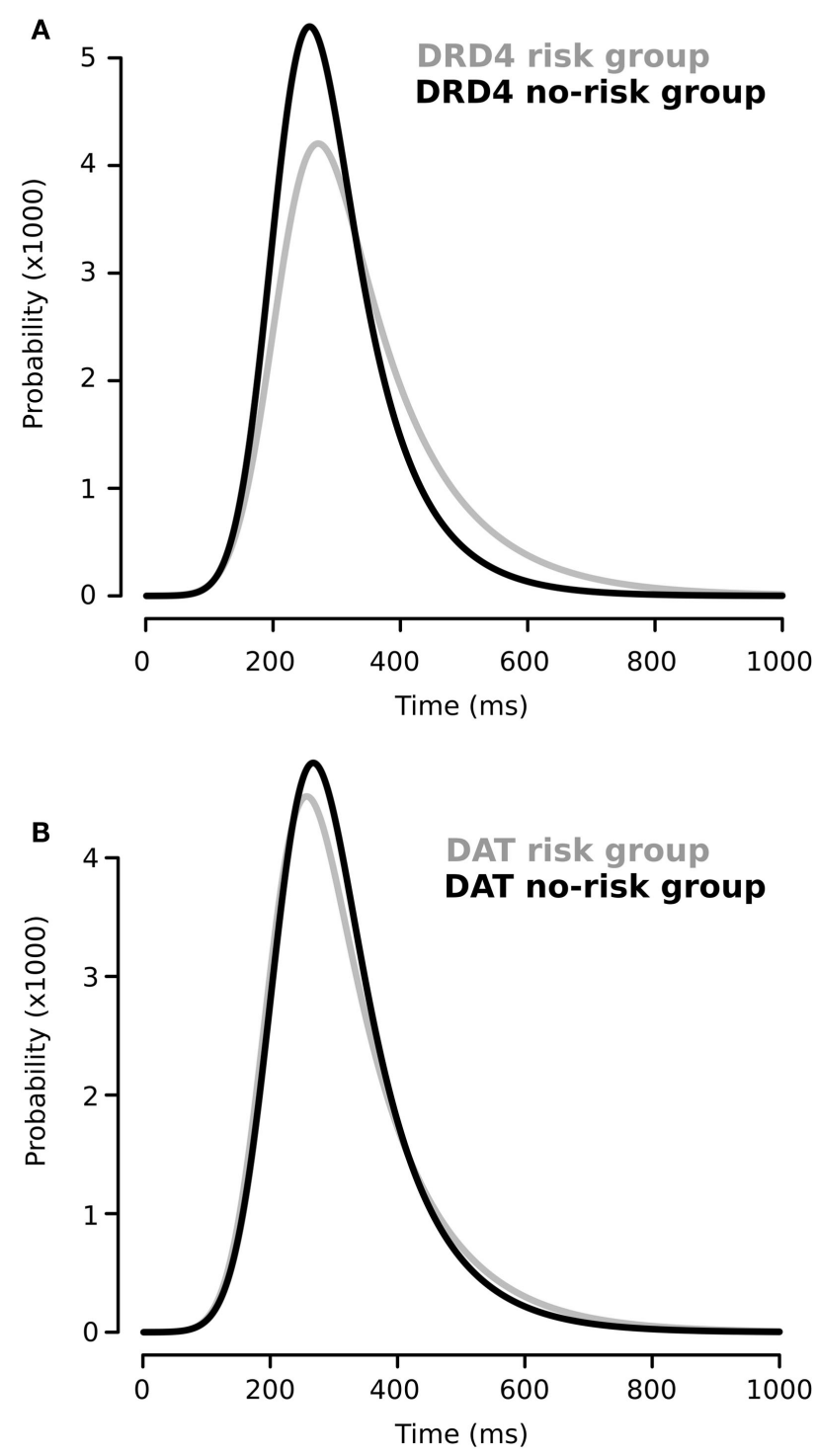

FIGURE 2 | Ex-Gaussian distributions for response times according to the presence/absence of previously described genetic risk variants for (A) DRD4 and (B) SCL6A3/DAT1. Black line: absence of risk alleles; gray line: presence of at least one risk allele (for a detailed description on group assignment criteria, please refer to Sections "Materials and Methods," and Sections "Group Comparisons").

found that $\tau$ values (representing the positive skew of the RT distribution) present the highest association with ADHD in tasks requiring limited executive control, while $\sigma$ became importantly correlated to $\mathrm{ADHD}$ when analyzing data from tasks requiring high executive/inhibition control $(11,22-24,26,27)$. Interestingly, the characteristics of the RT ex-Gaussian distribution among ADHD patients seems to be dependent on the task executive 
Table 2 | Ex-Gaussian parameters in subjects presenting "risk" versus subjects presenting "non-risk" alleles for DRD4 and SCL6A3/DAT1 genes.

\begin{tabular}{lcrccc}
\hline Gene & $\begin{array}{l}\text { Parameter } \\
\text { (ms) }\end{array}$ & $\begin{array}{l}\text { Risk } \\
\text { group }\end{array}$ & $\begin{array}{l}\text { Non-risk } \\
\text { group }\end{array}$ & $\begin{array}{l}\text { Unadjusted } \\
\boldsymbol{p} \text {-value }\end{array}$ & $\begin{array}{l}\text { Adjusted } \\
\boldsymbol{p} \text {-value }\end{array}$ \\
\hline DRD4 & $\mu$ & 212.2 & 209.1 & 0.96 & 0.56 \\
& $\sigma$ & 52.3 & 47.3 & 0.24 & 0.94 \\
& $\tau$ & 122.7 & 83.2 & $0.013^{*}$ & 0.14 \\
SCL6A3/DAT1 & $\mu$ & 214.8 & 201.8 & 0.48 & \\
& $\sigma$ & 51.2 & 47.2 & 0.92 & \\
& $\tau$ & 97.3 & 117.2 & 0.26 & \\
& & & & & \\
\hline
\end{tabular}

*Indicates a significance level of 0.05 or less.

a Unadjusted p-value was obtained by means of Wilcoxon sum rank test.

${ }^{b}$ Presented $p$-values are adjusted by age, sex, and clinical status (ADHD, healthy sibs, and/or TD children).

Table 3 | Estimated effects for all co-variates included in the linear regression models adjusted for $\mu, \sigma$, and $\tau$.

\begin{tabular}{|c|c|c|c|c|c|}
\hline $\begin{array}{l}\text { Model } \\
\text { for... }\end{array}$ & $\begin{array}{l}\text { Independent } \\
\text { variable }\end{array}$ & $\begin{array}{l}\text { Estimated } \\
\text { effect }\end{array}$ & SE & $p$-value & Effect-size, $r$ \\
\hline \multirow[t]{5}{*}{$\mu$} & Genotype & -23.75 & 21.98 & 0.29 & \\
\hline & Clinical status & 11.52 & 22.45 & 0.61 & \\
\hline & Age & -5.73 & 3.81 & 0.17 & \\
\hline & Sex & -19.73 & 20.23 & 0.34 & \\
\hline & $\begin{array}{l}\text { Genotype } \times \text { clinical } \\
\text { status }\end{array}$ & 24.97 & 28.76 & 0.39 & \\
\hline \multirow[t]{5}{*}{$\sigma$} & Genotype & -6.17 & 10.61 & 0.56 & \\
\hline & Clinical status & 1.5 & 10.83 & 0.89 & \\
\hline & Age & -3.35 & 1.84 & 0.07 & \\
\hline & Sex & -9.04 & 9.76 & 0.36 & \\
\hline & $\begin{array}{l}\text { Genotype } \times \text { clinical } \\
\text { status }\end{array}$ & 11.66 & 13.87 & 0.4 & \\
\hline \multirow[t]{5}{*}{$\tau$} & Genotype & -11.87 & 16.01 & 0.47 & \\
\hline & Clinical status & 4.78 & 16.36 & 0.77 & \\
\hline & Age & -5.79 & 2.78 & $0.05^{*}$ & 0.34 \\
\hline & Sex & -26.21 & 14.74 & 0.09 & \\
\hline & $\begin{array}{l}\text { Genotype } \times \text { clinical } \\
\text { status }\end{array}$ & 54.71 & 20.96 & $0.015^{*}$ & 0.41 \\
\hline
\end{tabular}

*Indicates a significance level of 0.05 or less.

control requirements, by one hand, and on the task duration, by the other hand, since some studies applying shorter tasks (3-7 min) have failed to find higher $\tau$ component on the RT data of ADHD patients. In the same line, the duration of the inter-stimulus interval can change the cognitive process measure in the Go/No-Go task (42). A recent meta-analysis shows that long inter-stimuli intervals are more sensitive to induce low inattentive responses (43). Thus, these findings suggest that both, the rate of stimuli presentation and the duration of the task, influence the amount of low RTs generated by attentional lapses.

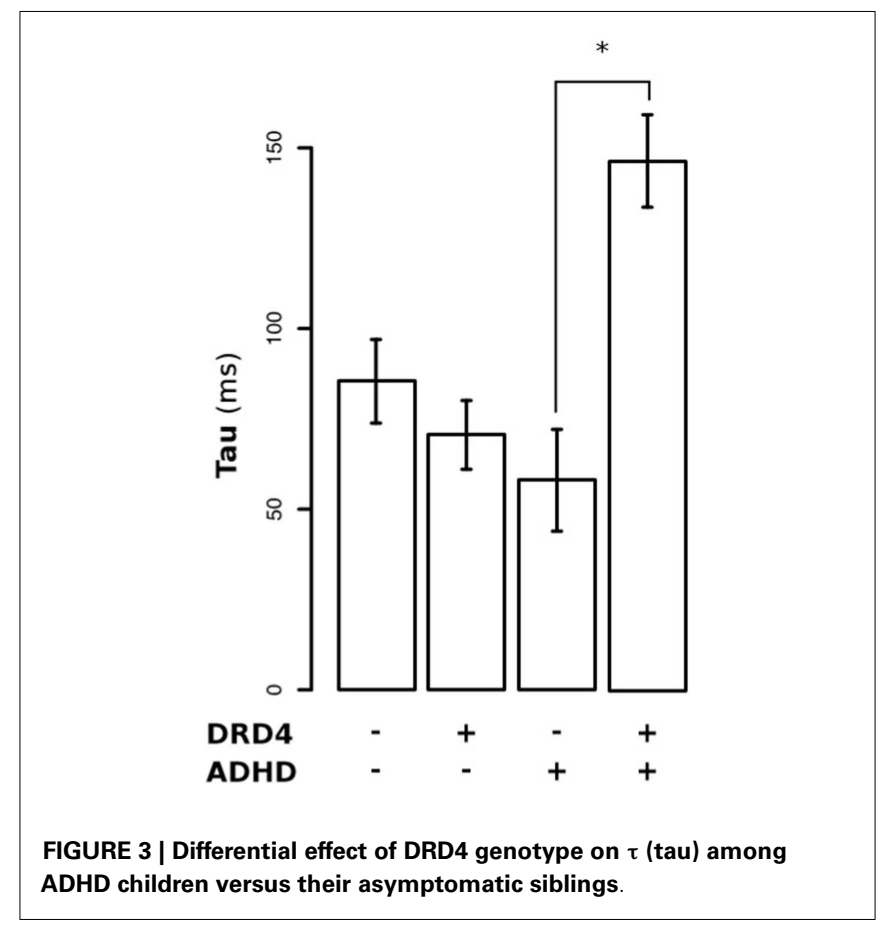

The Go-NoGo task used in this study demands high levels of executive/inhibition control due to extended task duration (about $8 \mathrm{~min}$ ), low probability for the NoGo stimuli (10\%), and shot inter-stimuli interval $(1000 \mathrm{~ms})$. In these conditions, the $\mu$ and $\sigma$ components obtained after fitting ex-Gaussian curves on the RT data from ADHD patients, healthy siblings, and TD children without family history of ADHD, showed the stair-like distribution expected for endophenotypes. This finding suggests that not only ADHD children but also ADHD healthy relatives (or at least a subpopulation of them) may present alterations in response preparation and motor timing. By contrast, our experimental conditions are not particularly conducive to the appearance of attentional lapses. Hence, it was expected that we failed to find the aforementioned distribution in the case of $\tau$ component, due to our short inter-trial periods. Nonetheless, the second part of our analysis showed a statistically significant effect of the "genotype" $\times$ "clinical status" interaction for DRD4 on $\tau(p$-value $=0.014)$. This result suggests that - in the context of a relatively long and high demanding inhibition task - attentional lapses may be predominately expressed on those ADHD patients carrying at least one copy of the $7 \mathrm{R}$ or $2 \mathrm{R}$ VNTR variants of DRD4. This finding is in accordance with other works, which associate DRD4 with severity of ADHD symptoms of impulsivity (44) and inattention (45). Thus, the presence of the risk alleles of DRD4 could reflect an eventual severity mark within the ADHD group that requires further study.

Although promising, our results require further analysis and replication. Our small sample size importantly limits the statistical power of the present study. Our inclusion criteria limit the generalization of our results only to patients presenting good response to stimulant medication. 


\section{CONCLUSION}

Together, our results suggest that intra-individual variability is suitable as an endophenotype for ADHD. Interestingly, under our conditions of high requirements of executive/inhibitory control, only the $\sigma$ component of variability presented the theoretical "stair-like distribution" for endophenotypes, while only $\tau$ (tau) seemed to be affected by the DRD4-genotype. Overall, our results emphasize the advantages of introducing new statistical methods oriented to reach a dynamic and fine-grain characterization of performance measures obtained from well-validated tasks in the analysis of new candidate-endophenotypes in psychiatric disorders. New studies are needed in order to confirm our results under different attention and inhibitory loads.

\section{AUTHOR CONTRIBUTIONS}

Study/experiments design: Marcela Patricia Henríquez-Henríquez, Francisco Aboitiz, and Francisco Rothhammer. Experiment execution: Marcela Patricia Henríquez-Henríquez, and Francisco Javier Zamorano. Genotyping: Hugo Henríquez and Francisco Rothhammer. Data analysis/statistical analysis: Marcela Patricia Henríquez-Henríquez and Pablo Billeke. Interpretation of results and discussion: Marcela Patricia Henríquez-Henríquez, Pablo Billeke, Francisco Aboitiz, and Francisco Rothhammer. Contributed reagents/materials/analysis tools: Marcela Patricia Henríquez-Henríquez, Pablo Billeke, Hugo Henríquez, and Francisco Rothhammer. Elaboration of the manuscript: Marcela Patricia Henríquez-Henríquez, Pablo Billeke, and Francisco Aboitiz. Critic lecture of the manuscript: Marcela Patricia HenríquezHenríquez, Pablo Billeke, Francisco Javier Zamorano, Francisco Rothhammer, and Francisco Aboitiz.

\section{ACKNOWLEDGMENTS}

This work was financed by the projects 1080219 and 1120412, from the National Fund for Science and Technology, FONDECYT, Chile; by Project "Anillo en Complejidad Social" (SOC-1101 to Pablo Billeke and Francisco Javier Zamorano), and by the Millenium Center for the Neuroscience of Memory, Chile, NC10-001-F.

\section{REFERENCES}

1. American Psychiatric Association E. Diagnostic and Statistical Manual of Mental Disorders: DSM-IV-TR ${ }^{\circledR}$. Washington, DC: American Psychiatric Publishing (2000).

2. World Health Organization. The ICD-10 Classification of Mental and Behavioural Disorders: Diagnostic Criteria for Research. Geneva: World Health Organization (1993).

3. Castellanos FX, Tannock R. Neuroscience of attention-deficit/hyperactivity disorder: the search for endophenotypes. Nat Rev Neurosci (2002) 3:617-28. doi:10.1038/nrn896

4. Gottesman II, Gould TD. The endophenotype concept in psychiatry: etymology and strategic intentions. Am J Psychiatry (2003) 160:636-45. doi:10.1176/appi. ajp.160.4.636

5. Levy Y, Ebstein RP. Research review: crossing syndrome boundaries in the search for brain endophenotypes. J Child Psychol Psychiatry (2009) 50:657-68. doi:10.1111/j.1469-7610.2008.01986.x

6. Gonzalez-Burgos G, Lewis DA. GABA neurons and the mechanisms of network oscillations: implications for understanding cortical dysfunction in schizophrenia. Schizophr Bull (2008) 34:944-61. doi:10.1093/schbul/sbn070

7. Gonzalez-Burgos G, Rotaru DC, Zaitsev AV, Povysheva NV, Lewis DA. GABA transporter GAT1 prevents spillover at proximal and distal GABA synapses onto primate prefrontal cortex neurons. J Neurophysiol (2009) 101:533-47. doi:10.1152/jn.91161.2008
8. Hall MH, Smoller JW. A new role for endophenotypes in the GWAS era: functional characterization of risk variants. Harv Rev Psychiatry (2010) 18:67-74. doi:10.3109/10673220903523532

9. Aase H, Meyer A, Sagvolden T. Moment-to-moment dynamics of ADHD behaviour in South African children. Behav Brain Funct (2006) 2:11. doi:10.1186/ 1744-9081-2-11

10. Russell VA, Oades RD, Tannock R, Killeen PR, Auerbach JG, Johansen EB, et al. Response variability in attention-deficit/hyperactivity disorder: a neuronal and glial energetics hypothesis. Behav Brain Funct (2006) 2:30. doi:10.1186/17449081-2-30

11. Buzy WM, Medoff DR, Schweitzer JB. Intra-individual variability among children with ADHD on a working memory task: an ex-Gaussian approach. Child Neuropsychol (2009) 15:441-59. doi:10.1080/09297040802646991

12. Klein C, Wendling K, Huettner P, Ruder H, Peper M. Intra-subject variability in attention-deficit hyperactivity disorder. Biol Psychiatry (2006) 60:1088-97. doi:10.1016/j.biopsych.2006.04.003

13. Johnson KA, Kelly SP, Bellgrove MA, Barry E, Cox M, Gill M, et al. Response variability in attention deficit hyperactivity disorder: evidence for neuropsychological heterogeneity. Neuropsychologia (2007) 45:630-8. doi:10.1016/j. neuropsychologia.2006.03.034

14. Uebel H, Albrecht B, Asherson P, Borger NA, Butler L, Chen W, et al. Performance variability, impulsivity errors and the impact of incentives as genderindependent endophenotypes for ADHD. J Child Psychol Psychiatry (2010) 51:210-8. doi:10.1111/j.1469-7610.2009.02139.x

15. Andreou P, Neale BM, Chen W, Christiansen H, Gabriels I, Heise A, et al. Reaction time performance in ADHD: improvement under fast-incentive condition and familial effects. Psychol Med (2007) 37:1703-15. doi:10.1017/ S0033291707000815

16. Epstein JN, Langberg JM, Rosen PJ, Graham A, Narad ME, Antonini TN, et al. Evidence for higher reaction time variability for children with $\mathrm{ADHD}$ on a range of cognitive tasks including reward and event rate manipulations. Neuropsychology (2011) 25:427-41. doi:10.1037/a0022155

17. Gould TD, Gottesman II. Psychiatric endophenotypes and the development of valid animal models. Genes Brain Behav (2006) 5:113-9. doi:10.1111/j.1601183X.2005.00186.x

18. Nigg JT, Blaskey LG, Stawicki JA, Sachek J. Evaluating the endophenotype model of ADHD neuropsychological deficit: results for parents and siblings of children with ADHD combined and inattentive subtypes. J Abnorm Psychol (2004) 113:614-25. doi:10.1037/0021-843X.113.4.614

19. Bidwell LC, Willcutt EG, Defries JC, Pennington BF. Testing for neuropsychological endophenotypes in siblings discordant for attention-deficit/ hyperactivity disorder. Biol Psychiatry (2007) 62:991-8. doi:10.1016/j.biopsych. 2007.04.003

20. Kuntsi J, Stevenson J. Psychological mechanisms in hyperactivity: II. The role of genetic factors. J Child Psychol Psychiatry (2001) 42:211-9. doi:10.1111/14697610.00712

21. Tamm L, Narad ME, Antonini TN, O’brien KM, Hawk LW Jr, Epstein JN. Reaction time variability in ADHD: a review. Neurotherapeutics (2012) 9:500-8. doi:10.1007/s13311-012-0138-5

22. Leth-Steensen C, Elbaz ZK, Douglas VI. Mean response times, variability, and skew in the responding of $\mathrm{ADHD}$ children: a response time distributional approach. Acta Psychol (Amst) (2000) 104:167-90. doi:10.1016/S0001-6918(00) 00019-6

23. Hervey AS, Epstein JN, Curry JF, Tonev S, Eugene Arnold L, Keith Conners $\mathrm{C}$, et al. Reaction time distribution analysis of neuropsychological performance in an ADHD sample. Child Neuropsychol (2006) 12:125-40. doi:10.1080/ 09297040500499081

24. Geurts HM, Grasman RP, Verte S, Oosterlaan J, Roeyers H, Van Kammen $\mathrm{SM}$, et al. Intra-individual variability in ADHD, autism spectrum disorders and Tourette's syndrome. Neuropsychologia (2008) 46:3030-41. doi:10.1016/j. neuropsychologia.2008.06.013

25. Vaurio RG, Simmonds DJ, Mostofsky SH. Increased intra-individual reaction time variability in attention-deficit/hyperactivity disorder across response inhibition tasks with different cognitive demands. Neuropsychologia (2009) 47:2389-96. doi:10.1016/j.neuropsychologia.2009.01.022

26. Gooch D, Snowling MJ, Hulme C. Reaction time variability in children with ADHD symptoms and/or dyslexia. Dev Neuropsychol (2012) 37:453-72. doi:10.1080/87565641.2011.650809 
27. Borella E, De Ribaupierre A, Cornoldi C, Chicherio C. Beyond interference control impairment in ADHD: evidence from increased intraindividual variability in the color-stroop test. Child Neuropsychol (2013) 19:495-515. doi:10.1080/09297049.2012.696603

28. Bellgrove MA, Hawi Z, Kirley A, Gill M, Robertson IH. Dissecting the attention deficit hyperactivity disorder (ADHD) phenotype: sustained attention, response variability and spatial attentional asymmetries in relation to dopamine transporter (DAT1) genotype. Neuropsychologia (2005) 43:1847-57. doi:10.1016/j. neuropsychologia.2005.03.011

29. Bellgrove MA, Hawi Z, Lowe N, Kirley A, Robertson IH, Gill M. DRD4 gene variants and sustained attention in attention deficit hyperactivity disorder (ADHD): effects of associated alleles at the VNTR and -521 SNP. Am J Med Genet B Neuropsychiatr Genet (2005) 136B:81-6. doi:10.1002/ajmg.b.30193

30. MacDonald SW, Cervenka S, Farde L, Nyberg L, Backman L. Extrastriatal dopamine D2 receptor binding modulates intraindividual variability in episodic recognition and executive functioning. Neuropsychologia (2009) 47:2299-304. doi:10.1016/j.neuropsychologia.2009.01.016

31. Carrasco X, Rothhammer P, Moraga M, Henriquez H, Chakraborty R, Aboitiz F, et al. Genotypic interaction between DRD4 and DAT1 loci is a high risk factor for attention-deficit/hyperactivity disorder in Chilean families. Am J Med Genet B Neuropsychiatr Genet (2006) 141B:51-4. doi:10.1002/ajmg.b.30259

32. American Psychiatric Association. Diagnostic and Statistical Manual of Mental Disorders, 4th Edition, Text Revision (DSM-IV-TR). Washington, DC: American Psychiatric Publishing (2000).

33. Rowe KS, Rowe KJ. Norms for parental ratings on Conners' abbreviated parentteacher questionnaire: implications for the design of behavioral rating inventories and analyses of data derived from them. J Abnorm Child Psychol (1997) 25:425-51. doi:10.1023/A:1022678013979

34. Lichter JB, Barr CL, Kennedy JL, Van Tol HH, Kidd KK, Livak KJ. A hypervariable segment in the human dopamine receptor D4 (DRD4) gene. Hum Mol Genet (1993) 2:767-73. doi:10.1093/hmg/2.6.767

35. Cook EH Jr, Stein MA, Krasowski MD, Cox NJ, Olkon DM, Kieffer JE, et al. Association of attention-deficit disorder and the dopamine transporter gene. Am J Hum Genet (1995) 56:993-8.

36. Henríquez Henríquez M, Villarroel L, Henríquez H, Zamorano F, Rothhammer F, Aboitiz F. Intratask variability as a correlate for DRD4 and SLC6A3 variants: a pilot study in ADHD. J Atten Disord (2012). doi:10.1177/1087054712455844

37. Yssaad-Fesselier R, Knoblauch K. Modeling psychometric functions in R. Behav Res Methods (2006) 38:28-41. doi:10.3758/BF03192747

38. Faraone SV, Doyle AE, Mick E, Biederman J. Meta-analysis of the association between the 7-repeat allele of the dopamine $\mathrm{D}(4)$ receptor gene and attention deficit hyperactivity disorder. Am J Psychiatry (2001) 158:1052-7. doi:10.1176/appi.ajp.158.7.1052

39. Manor I, Tyano S, Eisenberg J, Bachner-Melman R, Kotler M, Ebstein RP. The short DRD4 repeats confer risk to attention deficit hyperactivity disorder in a family-based design and impair performance on a continuous performance test (TOVA). Mol Psychiatry (2002) 7:790-4. doi:10.1038/sj.mp.4001078

40. Leung PW, Lee CC, Hung SF, Ho TP, Tang CP, Kwong SL, et al. Dopamine receptor D4 (DRD4) gene in Han Chinese children with attention-deficit/ hyperactivity disorder (ADHD): increased prevalence of the 2-repeat allele. Am J Med Genet B Neuropsychiatr Genet (2005) 133B:54-6. doi:10.1002/ajmg. b.30129

41. Asghari V, Sanyal S, Buchwaldt S, Paterson A, Jovanovic V, Van Tol HH. Modulation of intracellular cyclic AMP levels by different human dopamine D4 receptor variants. J Neurochem (1995) 65:1157-65. doi:10.1046/j.1471-4159.1995. 65031157.x

42. Zamorano F, Billeke P, Hurtado JM, Lopez V, Carrasco X, Ossandon T, et al. Temporal constraints of behavioral inhibition: relevance of inter-stimulus interval in a Go-Nogo task. PLoS One (2014) 9:e87232. doi:10.1371/journal.pone.0087232

43. Metin B, Roeyers H, Wiersema JR, Van Der Meere J, Sonuga-Barke E. A metaanalytic study of event rate effects on Go/No-Go performance in attentiondeficit/hyperactivity disorder. Biol Psychiatry (2012) 72:990-6. doi:10.1016/j. biopsych.2012.08.023

44. Sharp SI, Mcquillin A, Gurling HM. Genetics of attention-deficit hyperactivity disorder (ADHD). Neuropharmacology (2009) 57:590-600. doi:10.1016/j. neuropharm.2009.08.011

45. Gizer IR, Waldman ID, Abramowitz A, Barr CL, Feng Y, Wigg KG, et al. Relations between multi-informant assessments of ADHD symptoms, DAT1, and DRD4. J Abnorm Psychol (2008) 117:869-80. doi:10.1037/a0013297

Conflict of Interest Statement: The authors declare that the research was conducted in the absence of any commercial or financial relationships that could be construed as a potential conflict of interest.

Received: 04 November 2014; paper pending published: 26 November 2014; accepted: 20 December 2014; published online: 12 January 2015.

Citation: Henríquez-Henríquez MP, Billeke P, Henríquez H, Zamorano FJ, Rothhammer $F$ and Aboitiz $F$ (2015) Intra-individual response variability assessed by ex-Gaussian analysis may be a new endophenotype for attention-deficit/hyperactivity disorder. Front. Psychiatry 5:197. doi: 10.3389/fpsyt.2014.00197

This article was submitted to Child and Neurodevelopmental Psychiatry, a section of the journal Frontiers in Psychiatry.

Copyright (c) 2015 Henríquez-Henríquez, Billeke, Henríquez, Zamorano, Rothhammer and Aboitiz. This is an open-access article distributed under the terms of the Creative Commons Attribution License (CC BY). The use, distribution or reproduction in other forums is permitted, provided the original author(s) or licensor are credited and that the original publication in this journal is cited, in accordance with accepted academic practice. No use, distribution or reproduction is permitted which does not comply with these terms. 